\title{
Economic Burden of Epidermolysis Bullosa Disease in Iran
}

\author{
Siamak Farokh Forghani ${ }^{1}$, Reza Jahangiri ${ }^{2}$, Farzaneh Ghasemi ${ }^{3}$, Reza Shirani ${ }^{4}$, Hosein Bagheri ${ }^{5}$, Razieh Mahmoodi ${ }^{3}$, \\ Saeed Bagheri Faradonbeh ${ }^{2} *$ (1)
}

Received: 26 Oct 2020

Published: 3 Nov 2021

\begin{abstract}
Background: Epidermolysis bullosa (EB) is prevalent in Iran and incurs direct and indirect costs on the health care system and the patient.Therefore, this study was conducted to estimate the economic burden of this disease in Iran.

Methods: This study includes all patients with EB disease who had been referred to the medical centers of Iran in 2019-2020 for treatment of their disease, especially Hazrat Fatimah Hospital and their medical records are available in Iran EB Patients Association (IEBPA). In estimating the economic burden of diseases and costing studies, we calculated the average direct and indirect costs for a patient and used it to estimate the costs of the patient population. In this study, a prevalence approach was used to calculate the economic burden of this disease. For this purpose, the existing cases of the disease in 2019-2020 were calculated. The data collected from the questionnaires that were completed through interviews with patients as well as the data extracted from the review of their files were entered into Excel software and analyzed.

Results: The total direct medical costs of direct non-medical and indirect are equal to 7.319.428.315 \& 5.390.440.775 and 45.875.654.514 Rials respectively, and the total economic burden of the disease and the average of each patient is 58.585.514.604 and 155.890 .789 respectively.

Conclusion: High indirect costs, especially informal care, represent the socio-economic burden of this disease and constitute more than half of the total indirect costs. Therefore, the obvious gap and hidden social costs of this disease are related to health policymakers, especially when they want to study the impact of this disease on different income classes of families.
\end{abstract}

Keywords: Economic Burden, Epidermolysis Bullosa, EB, Iran

Conflicts of Interest: None declared

Funding:Iran University of Medical Sciences

*This work has been published underCC BY-NC-SA 1.0 license.

Copyright $₫$ Iran University of Medical Sciences

Cite this article as: Forghani SF, Jahangiri R, Ghasemi F, Shirani R, Bagheri H, Mahmoodi R, Bagheri Faradonbeh S. Economic Burden of Epidermolysis Bullosa Disease in Iran.Med J Islam Repub Iran. 2021(3 Nov);35:146. https://doi.org/10.47176/mjiri.3;5.146

\section{Introduction}

Epidermolysis bullosa (EB) is a rare skin disease and an inherited disorder. In this disease, there are connective tissue disorders that are the result of the defective connection of its inner layers, which is fragile. The result is that the top layer of skin does not protect the bottom layer and blister forms where the two layers are separated. The disease is divided into four types based on the affected skin: simplex, junctional, dystrophic, and Kindler syndrome (1,

Corresponding author: Dr Saeed Bagheri Faradonbeh, bagheri.s@iums.ac.ir

${ }^{1 .}$ Burn Research Center, Iran University of Medical Sciences, Tehran, Iran

2. Health Management and Economics Research Center, Iran University of Medical Sciences, Tehran, Iran

3. Department of Health Economics, School of Health Management and Information Sciences, Iran University of Medical Sciences, Tehran, Iran

4. Department of Public Health Economics, School of Public Health, Shahrekord University of Medical Sciences, Shahrekord, Iran

5chool of Health Management and Information Sciences, Iran University of Medical Sciences, Tehran, Iran
2).

Epidermolysis bullosa simplex (EBS) usually is inherited in an autosomal dominant manner, meaning that a defective gene inherited from a parent is enough to develop the condition. Junctional Epidermolysis Bullosa (JEB) develops when the defective gene is inherited from both parents who are EB carriers but do not show any symptoms themselves. Dystrophic Epidermolysis Bullosa

$\uparrow$ What is "already known" in this topic:

Estimating the economic burden of the EB disease plays an important role in informing decisions about reimbursement, estimating the costs of the disease, and producing scientific evidence for policy decisions.

\section{$\rightarrow$ What this article adds:}

This study indicated that High indirect costs, especially informal care, represent the socio-economic burden of this disease and constitute more than half of the total indirect costs. 
(DEB) is a form of inherited EB characterized by cutaneous and mucosal fragility resulting in blisters and superficial ulcerations that develop below the lamina dense of the cutaneous basement membrane and that heal with significant scarring and milia formation. Kindler syndrome is a rare type of EB, which is a group of genetic conditions that cause the skin to be very fragile and to blister easily (3-5).

The burden of EB disease is high and severely affects the quality of life. The treatment of this disease so far is supportive and in order to heal the wound and resolve the complications. In recent years, a number of experimental treatments for EB have been investigated, most of which are dedicated to EB dystrophy (6). the prevalence of the disease is 5 per 100,000 live births, regardless of gender or race (7). In the European Union, the prevalence is 1 to 2 per 100,000 people (8). Disease cost studies are a systematic quantification of the economic burden of disease on the individual and society, which examines the direct impact of disease on the budget of the health system and the indirect costs associated with loss of productivity or care (9). The average annual cost per patient in 2012 for patients in Bulgaria, France, Germany, Hungary, Italy, Spain, Sweden and the United Kingdom was 17671, 14931, 46116, 9809, 49233, 43177, 9509 and $19758 \mathrm{Eu}-$ ros, respectively (8).

The economic impact of health conditions in general and non-communicable diseases in particular is of special importance and interest to policymakers. Faced with increases in the prevalence of chronic conditions, governments, and other stakeholders may wish to enact policies that are effective at reducing disease incidence and its consequences (10). Studying the cost of disease measures the socio-economic burden of disease and helps to prioritize and justify health care and prevention policies (11). Studying the cost of disease by comparing the financial burden can show which intervention reduces the cost and thus leads to a change in the distribution of investments. The government can estimate the financial impact of the disease on the public budget for optimal resource allocation, and pharmaceutical companies can invest in research and development of the disease (12-14). Also knowing the costs of a disease can help policymakers decide which disease should be considered as the first disease by prevention with health care policies. In addition, these studies can indicate which treatments for the disease can be helpful in reducing the economic burden of the disease (1517).

According to the results of other studies, $26 \%$ of patients with EB spent more than $\$ 1000$ per month on wound care supplies (18), and average annual costs in these patients varied from country to country and ranged from $€ 9509$ to $€ 49,233$ and estimated that direct medical costs ranged from $€ 419$ to $€ 10,688$; direct non-medical costs ranged from $€ 7449$ to $€ 37,451$ and labor productivity losses ranged from $€ 0$ to $€ 7259$ (8). and most of the rare diseases examined are associated with significant economic burdens, both direct and indirect (9).

Estimating the economic burden of the EB disease plays an important role in informing decisions about reim- bursement, estimating the costs of the disease, producing scientific evidence for policy decisions, and finally, the optimal allocation of limited health financial resources in the field of economics of rare diseases (19-21). Due to the fact that this disease is also prevalent in Iran and incurs direct and indirect costs on the health care system and the patient and no study on the economic burden of this disease has been conducted in the country so far, so the implementation of this research seems necessary. So the aim of this study was conducted to estimate the economic burden of this disease in Iran.

\section{Methods}

This is a cross-sectional study in which all biographical information and information about the direct and indirect costs of EB disease in 2019-2020 from 2015-2018 were collected.There was no time interval between diagnosis and data collection because the disease is chronic and the minimum criterion for patients to be included in the study was that at least more than one year has passed since their illness. The study population is all patients with EB in the whole country. The method of data collection is numerical and the research population is people with EB whose files are available in the Iran EB Patients Association (IEBPA) and have been treated in Hazrat Fatimah Hospital. The choice of this hospital as a research environment has been due to performing all treatment processes related to different stages of EB, including diagnosis, surgery, radiotherapy and chemotherapy in this Center.

In this study, the average economic burden of EB is described in terms of cost at different stages of the disease, in terms of Rial value. And because the information obtained can be used in policymaking and resource allocation in the health sector, it is considered a practical study. In estimating the economic burden of diseases and costing studies, we calculated the average direct and indirect costs for a patient and used it to estimate the costs of the patient population. Due to the fact that the treatment protocols and tariffs for medical services are the same throughout the country, by selecting the patients referred to this center, the average cost per patient was obtained, which could be generalized to the community of patients with this disease.

In general, there are two approaches to calculate the economic burden of diseases: the incidence-based approach and the prevalence-based approach. In the incidence-based approach, new cases of the disease are considered in that particular period; but in the prevalencebased approach, the economic burden associated with existing cases of the disease over a given period is estimated. The prevalence approach has the advantage of taking into account the total annual cost of health care, especially for chronic diseases such as diabetes, heart disease and cancer that require long-term treatment (22-24). Therefore, considering this advantage, in this study, we used a prevalence-based approach to calculate the economic burden of Epidermolysis Bullosa. For this purpose, the existing cases of the disease in 2019-2020 were studied.

In the present study, the costs of the disease were calculated from a social perspective because in this approach, 
the real costs imposed on society are estimated. These costs include direct medical costs, non-medical direct costs and indirect costs of EB disease. Direct medical costs included the cost of visits, diagnosis, testing and diagnostic services, the cost of purchasing medication (both prescription and over-the-counter), hospitalization, and patient care. Non-medical direct costs are costs incurred in receiving treatment and diagnostic services for EB disease. Such costs include costs for travel, hotel accommodation costs, costs of food during travel and so forth. Indirect costs of a disease include lost productivity due to the disease. These costs are due to disability due to illness (absence from work) or due to premature death due to illness (25-28) in this study For patients who were children, the costs associated with the loss of economic production were calculated for the patient's parent or caregiver. Data were completed through questionnaires and interviews with patients and entered into Excel software and analyzed and patients and their families were assured that their information would remain confidential and would be used solely for this research.

\section{Results}

In this study, the files of 546 patients with EB were studied. First, the findings of demographic information of individuals are expressed, then the cost items of treatment of these patients are identified and the amount of each of them is discussed separately. Finally, the direct costs of medical and non-medical of patients and indirect costs of patients for the treatment of this disease are expressed.

\section{Basic patient characteristics}

Table 1 describes the Basic patient characteristics. As can be seen, $52 \%$ of patients are male, and $48 \%$ of them are female. In terms of age range, $49 \%$ of patients are in the range of 1 to 10 years, $26 \%$ are 11 to 20 years old, $15 \%$ are 21 to 30 years old and $9 \%$ are in the age range of more than 31 years. Also, in terms of education variables, $76 \%$ of them were illiterate, $20 \%$ were low literate and $4 \%$ had a university education. $91 \%$ of patients were unemployed, 3\% were employees and 5\% were self-employed. Regarding the length of hospital stay, $25 \%$ of them were hospitalized for 10 to 15 days, $28 \%$ for 16 to 20 days, $29 \%$ for 21 to 25 days and $17 \%$ for 26 to 30 days. Regarding the type of disease, $73 \%$ of patients had dystrophic, $11 \%$ junctional, $8 \%$ simplex, $2 \%$ kindler and $3 \%$ unknown. The last variable studied in this study was the residence of patients, $11 \%$ of whom lived in Tehran and $89 \%$ in other provinces.

\section{Direct medical costs}

Table 2 shows the dimensions of direct medical costs. As can be seen, the highest cost item is related to the cost of the inpatient bed, followed by the costs of the medical team and consumables; then there are operating room costs, other services, diagnostic services, ward medicines and nursing services costs.

\section{Direct non-medical costs}

Table 3 shows the direct non-medical costs. As can be seen, this type of expense in this study included travel, accommodation, and nutrition, which included 30, 62 and $8 \%$ of the expenses, respectively.

\begin{tabular}{|c|c|c|c|}
\hline Variable & & Frequently & Average $\pm \mathrm{SD}$ \\
\hline \multirow[t]{2}{*}{ Sex } & Male & $285(\% 52)$ & \\
\hline & Female & $261(\% 48)$ & \\
\hline \multirow[t]{4}{*}{ Age } & $1-10$ & $270(\% 49)$ & $19.74 \pm 5.23$ \\
\hline & $11-20$ & $144(\% 26)$ & \\
\hline & $21-30$ & $83(\% 15)$ & \\
\hline & $\geq 31$ & $49(\% 9)$ & \\
\hline \multirow[t]{3}{*}{ Education } & Illiterate & $414(\% 76)$ & \\
\hline & Low literate & $108(\% 20)$ & \\
\hline & University & $23(\% 4)$ & \\
\hline \multirow[t]{3}{*}{ Employment status } & Unemployed & $498(\% 91)$ & \\
\hline & Employee & $18(\% 3)$ & \\
\hline & Self-employed & $30(\% 5)$ & \\
\hline \multirow[t]{4}{*}{ Hospitalization } & $10-15$ & $137(\% 25)$ & $15.19 \pm 12.07$ \\
\hline & $16-20$ & $155(\% 28)$ & \\
\hline & $21-25$ & $160(\% 29)$ & \\
\hline & $26-30$ & $94(\% 17)$ & \\
\hline \multirow[t]{5}{*}{ Epidermolysis bullosa (EB) subtype } & Dystrophic & $398(\% 73)$ & \\
\hline & Junctional & $58(\% 11)$ & \\
\hline & Simplex & $42(\% 8)$ & \\
\hline & Kindler & $10(\% 2)$ & \\
\hline & Unkhown & $16(\% 3)$ & \\
\hline \multirow[t]{2}{*}{ Place of residence } & Tehran & $61(\% 11)$ & \\
\hline & Other provinces & $485(\% 89)$ & \\
\hline
\end{tabular}




\begin{tabular}{|c|c|c|c|c|}
\hline \multicolumn{5}{|c|}{ Table 2.Dimensions of direct Medical costs } \\
\hline Cost dimension & Average & Standard Deviation & Total Costs & Percent \\
\hline Inpatient bed days & 4.590 .192 & 277.405 & 2.562 .319 .750 & 35.01 \\
\hline Nursing services & 258.292 & 15.610 & 144.182 .279 & 1.97 \\
\hline Medical team & 3.243 .664 & 196.028 & 1.810 .666 .034 & 24.74 \\
\hline Operating room & 699.579 & 42.279 & 390.516 .460 & 5.34 \\
\hline Medicine & 445.775 & 26.940 & 248.838 .656 & 3.40 \\
\hline Consumables & 2.794 .965 & 168.912 & 1.560 .195 .015 & 21.32 \\
\hline Diagnostic services & 469.299 & 28.362 & 261.970 .456 & 3.58 \\
\hline Other services & 610.408 & 36.980 & 340.739 .365 & 4.66 \\
\hline Total & 13.112 .174 & & 7.319.428.315 & 100 \\
\hline \multicolumn{5}{|l|}{ Table 3.Direct non-medical costs } \\
\hline Cost dimension & Average & Standard Deviation & Total Costs & Percent \\
\hline Travel & 2.830 .250 & 172.281 & 1.579 .829 .500 & 30 \\
\hline Accommodation & 6.025 .050 & 369.476 & 3.363 .692 .400 & 62 \\
\hline Nutrition & 800.563 & 49.171 & 446.918 .875 & 8 \\
\hline Total & 9.655 .863 & & 5.390 .440 .775 & 100 \\
\hline \multicolumn{5}{|c|}{ Table 4.Dimensions of patients' indirect costs } \\
\hline Cost dimension & & Standard Deviation & Total Costs & Percent \\
\hline Related to Patients & & 5.910 .293 & 16.062 .092 .046 & 35 \\
\hline Related to patients companions & & 555.668 & 3.569 .353 .788 & 8 \\
\hline Informal care & & 4.849 .471 & 26.244 .199 .680 & 57 \\
\hline Total & & & 45.875 .645 .514 & 100 \\
\hline \multicolumn{5}{|c|}{ Table 5. The economic burden of the disease } \\
\hline Cost dimension & & & & Percent \\
\hline Medical Direct costs & & & 28.315 & 12 \\
\hline Non-medical Direct costs & & & 40.775 & 10 \\
\hline Indirect costs & & 45. & 45.514 & 78 \\
\hline Economic burden of disease & & 58. & 14.604 & 100 \\
\hline
\end{tabular}

\section{Indirect costs}

Table 4 shows the dimensions of patients' indirect costs. As can be seen, $35 \%$ of the total indirect costs are related to patients, $8 \%$ are related to patient companions, and $57 \%$ are related to informal care for patients.

\section{The economic burden of disease}

Table 5 shows the economic burden of the disease. According to this table, the total costs of direct medical, direct non-medical, and indirect are equal to 7.319.428.315, 5.390.440.775, and 45.875.645.514 Rials, respectively. And the economic burden of the disease in total and average of each patient is equal to 58.585.514.604 and 155.890.789 Rials, respectively. Also, according to this study, $12 \%$ of the total costs are related to direct medical costs, $10 \%$ non-medical direct costs and $78 \%$ are related to indirect costs.

\section{Discussion}

The aim of this study was to estimate the economic burden of epidermolysis bullosa in Iran. The present study highlights the importance of studying the economic implications of EB and interpreting the results at the national level. The results of our analysis provide insights into the distribution of EB costs and the impact of the disease on national health care costs. Therefore, based on this goal, in this study, first the direct costs of treatment, then the direct non-medical and finally the indirect costs and economic burden of the disease were addressed. According to the results of this study, in the direct medical costs, the most costly items are related to the cost of hospital beds, then are the costs of the medical team and consumables, costs of the operating room, other services, diagnostic services, medicine and the costs of nursing services. Kyung Jeon et al. Concluded in their study that as skin lesions enlarge, the economic burden of the disease is more on dressing change, and since all participants in the study had skin lesions larger than $30 \%$, a large portion of the costs so their direct medical costs were related to changing the dressing also, based on their study and the results of the present study, side effects due to skin barrier disorders and insufficient immunity may increase "medical costs" in young patients (29). Also, according to Darragh Flannery et al. Study, out of the total direct medical costs related to this disease, the highest is related to the cost of hospital stay, medicine and wound care, other primary care and daily clinics or daily outpatient care, respectively (30) which is consistent with the results of our study.

Also, in the field of non-medical direct costs, this type of cost in this study included travel, patient accommodation and nutrition, which included 30,62 and $8 \%$ of the total costs, respectively. Also, for indirect costs, 35\% of the total indirect costs are related to patients, $8 \%$ are related to patient companions and $57 \%$ are related to informal care for patients. The high cost of informal care reflects the socioeconomic burden of the disease and accounts for more than half of all indirect costs. Therefore, the obvious gap and hidden social costs of this disease are related to health policymakers, especially when they want to study the impact of this disease on different income classes of families.

Also, according to this study, $12 \%$ of the total costs are 
related to direct medical costs, $10 \%$ are direct costs of non-medical and $78 \%$ are related to indirect costs and the economic burden of the disease in total and the average of each patient is equal to 58.585.514.604 and 155.890.789 Rials, respectively. According to a study by Aris Angelis et al. The average annual cost per patient in all countries is estimated at $€ 31,390$, of which $€ 5,646$ is direct medical costs $(18 \%)$ and $€ 23,483$ is direct non-medical costs (74.8\%) and 2261 Euros included indirect costs (7.2\%) (8). That in terms of direct medical costs, it is consistent with the results of our study and in other dimensions is not consistent, which could be due to differences in the system of providing services to these patients in different countries or different policies to treat these patients in this Countries. Also, Emily et al. In their study, they showed that the financial burden of EB in the United States is high and the US health care system needs the support of the federal budget for direct medical costs, especially bandaging(18). In another study, Aris Angelis et al. concluded that rare diseases, such as EB, have a high economic burden, and those indirect costs, along with loss of productivity, often approach or even exceed direct cost levels (9). Their results are consistent with the findings of the present study.

Our study has some strengths. First, this hospital is the only center that can treat these patients and almost all patients in Iran with EB are referred to this hospital and their information is recorded in Iran EB Patients Association (IEBPA), that all this information was used in this study. Secondly, the present study can help to understand the economic burden associated with $\mathrm{EB}$ and, in turn, better meet the needs of patients with the disease. On the other hand, the small sample size of this study can be a limitation, although the incidence of this disease is very low.

\section{Conclusion}

According to this study, $12 \%$ of the total costs are related to direct medical costs, $10 \%$ are direct costs of nonmedical and $78 \%$ are related to indirect costs, and the economic burden of the disease in total and the average of each patient is equal to 58.585.514.604 and 155.890.789 Rials,respectively. health Policymakers and planners need to be aware of the direct and indirect costs of this disease in order to decide on the correct allocation of resources. Therefore, policymakers should consider this issue in the macro policies of the health system and provide facilities with the cooperation of physicians for timely prevention and treatment of these patients.

\section{Acknowledgment}

This research has been supported by Iran University of Medical Sciences (code of ethics: IR.IUMS.REC.1398.068). The authors would like to thank Iran University of Medical Sciences and the Iran EB Patients Association (IEBPA) for supporting this study.

\section{Conflict of Interests}

The authors declare that they have no competing interests.

\section{References}

1. Fine JD, Hintner H. Life with epidermolysis bullosa (EB): Springer; 2009.

2. Fine JD, Eady RAJ, Bauer EA, Bauer JW, Bruckner-Tuderman L, Heagerty A, et al. The classification of inherited epidermolysis bullosa (EB): Report of the Third International Consensus Meeting on Diagnosis and Classification of EB. J Am Acad Dermatol. 2008;58(6):931-50.

3. Fine JD. Inherited epidermolysis bullosa. Orphanet $\mathrm{J}$ Rare Dis. 2010;5(1):1-17.

4. Yuen WY, Duipmans JC, Molenbuur B, Herpertz I, Mandema JM, Jonkman MF. Long-term follow-up of patients with Herlitz-type junctional epidermolysis bullosa. Br J Dermatol. 2012;167(2):374-82.

5. Sawamura D, Nakano H, Matsuzaki Y. Overview of epidermolysis bullosa. J Dermatol. 2010;37(3):214-9.

6. Has C. Molecular therapies for inherited epidermolysis bullosa. G Ital Dermatol Venereol. 2016;151(4):397-402.

7. NIH. Genetic home reference [2011 May 6th:[Available from: http://ghr.nlm.nih.gov/condition/epidermolysis-bullosa-simplex.

8. Angelis A, Kanavos P, López-Bastida J, Linertová R, Oliva-Moreno J, Serrano-Aguilar P, et al. Social/economic costs and health-related quality of life in patients with epidermolysis bullosa in Europe. Eur J Health Econ. 2016;17(1):31-42.

9. Angelis A, Tordrup D, Kanavos P. Socio-economic burden of rare diseases: a systematic review of cost of illness evidence. Health Policy. 2015;119(7):964-79.

10.Bloom DE, Chisholm D, Jané-Llopis E, Prettner K, Stein A, Feigl A. From burden to" best buys": reducing the economic impact of noncommunicable disease in low-and middle-income countries. Program Global Demog Aging; 2011.

11.Rice DP. Cost of illness studies: what is good about them? Inj Prev. 2000;6(3):177-9.

12.Chapel JM, Ritchey MD, Zhang D, Wang G. Prevalence and medical costs of chronic diseases among adult Medicaid beneficiaries. Am J Prev Med. 2017;53(6):S143-S54.

13.Rezapour A, Jahangiri R, Nikjoo S, Farabi H, Soleimanpour S. Economic Burden of Osteoporosis in the World: A Systematic Review. Med J Islam Repub Iran. 2020. 34:154

14.Arab-Zozani M, Moynihan RN, Pezeshki MZ. Shared decision making: How can it be helpful in reducing medical overuse due to medical misinformation mess? J Eval Clin Pract. 2020.

15.Oremus M, Aguilar SC. A systematic review to assess the policymaking relevance of dementia cost-of-illness studies in the US and Canada. Pharmacoeconomics. 2011;29(2):141-56.

16.Finkelstein E, Corso P. Cost-of-illness analyses for policy making: a cautionary tale of use and misuse. Taylor \& Francis; 2003.

17.Rezapour A, Tavakoli N, Akbar S, Hajahmadi M, Ameri H, Mohammadi R, et al. Medical therapy versus percutaneous coronary intervention in ischemic heart disease: A cost-effectiveness analysis. Med J Islam Repub Iran. 2020;34:155.

18.Gorell ES, Wolstencroft PW, de Souza MP, Murrell DF, Linos E, Tang JY. Financial burden of epidermolysis bullosa on patients in the United States. Pediatr Dermatol. 2020;37(6):1198-1201.

19.LaVeist TA, Gaskin D, Richard P. Estimating the economic burden of racial health inequalities in the United States. Int J Health Serv. 2011;41(2):231-8.

20.Henrard S, Devleesschauwer B, Beutels P, Callens M, De Smet F, Hermans $\mathrm{C}$, et al. The health and economic burden of haemophilia in Belgium: a rare, expensive and challenging disease. Orphanet J Rare Dis. 2014;9(1):39.

21.Cai X, Yang H, Genchev GZ, Lu H, Yu G. Analysis of economic burden and its associated factors of twenty-three rare diseases in Shanghai. Orphanet J Rare Dis. 2019;14(1):233.

22.Daroudi A, Zendehdel K, Nahvijou A, Zahmatkesh H, Akbarisari A. A review of methods for estimating economic burden of cancer. Hakim Res J. 2014;16(4)

23.Bloom DE, Chen S, Kuhn M, McGovern ME, Oxley L, Prettner K. The economic burden of chronic diseases: estimates and projections for China, Japan, and South Korea. J Econ Ageing. 2018:100163.

24. Habetha S, Bleich S, Weidenhammer J, Fegert JM. A prevalencebased approach to societal costs occurring in consequence of child abuse and neglect. Child Adolesc Psychiatry Ment Health. 2012;6(1):35.

25.Goates S, Du K, Braunschweig CA, Arensberg MB. Economic 
burden of disease-associated malnutrition at the state level. PloS One. 2016;11(9):e0161833.

26.Drucker AM, Wang AR, Qureshi AA. Research gaps in quality of life and economic burden of atopic dermatitis: the National Eczema Association burden of disease audit. JAMA Dermatol. 2016;152(8):873-4.

27.Daroudi R, Sari AA, Nahvijou A, Kalaghchi B, Najafi M, Zendehdel $\mathrm{K}$. The economic burden of breast cancer in Iran. Iran J Public Health. 2015;44(9):1225.

28.Ameri H, Safari H, Poder T. Exploring the consistency of the SF$6 \mathrm{Dv} 2$ in a breast cancer population. Expert Rev Pharmacoecon Outcomes Res. 2020:1-8.

29.Jeon IK, On HR, Kim S-C. Quality of life and economic burden in recessive dystrophic epidermolysis bullosa. Ann Dermatol. 2016;28(1):6-14.

30.Flannery D, Doyle C, Hickey S, Aherne F, Kennan A. Direct Costs of Epidermolysis Bullosa by Disease Severity. Acta Derm Venereol. 2020;100(6-7):1-2. 